Background: The long-term psychiatric sequelae of war service have not been assessed for Australian veterans in a cohort study.

Methods: An epidemiologic cohort study was conducted with a randomly selected sample of 1000 Australian Army Vietnam veterans who were first assessed in 1991-1993 and again in 2005-2006 using standardized psychiatric assessment instruments (SCID and Mississippi in 1991-1993 and CAPS and Composite International Diagnostic Interview in 2005-2006). Personal interviews were conducted across Australia.

Results: The lifetime prevalence of post-traumatic stress disorder (PTSD) had moved from approximately $20 \%$ in $1991-1993$ to more than $50 \%$ in $2005-2006$, with smaller prevalence of current (1 month) PTSD. This was often accompanied by long-term use of psychoactive medications, while use of clinical PTSD programmes was also helpful. The course of PTSD was found to be variable: for some, symptoms were evident soon after return, for others, later life events acted as triggers for more recent coping breakdown. Alcohol overuse remains veterans' biggest problem.

Conclusion: PTSD is a chronic condition that is clearly evident in former soldiers and whose life course is variable.

\section{Identifying affective markers of ADHD and comorbid Conduct Disorder}

\section{Palmer', L Williams ${ }^{1,2,3}$, E Gordon ${ }^{1,4}$, D Hermens ${ }^{1,4}$}

${ }^{1}$ The Brain Dynamics Centre; ${ }^{2}$ Westmead Millennium Institute; ${ }^{3}$ Western Clinical School, The University of Sydney; and 'Brain Resource Company, Sydney, Australia

Objective: Conduct disorder (CD) encompasses children and adolescents who show extreme antisocial behaviour and is commonly allied with attention deficit hyperactivity disorder (ADHD). This study focuses on the markers of facial emotion recognition, which discriminate ADHD from combined ADHD and CD, as well as healthy controls.

Method: Children and adolescents with ADHD, with and without comorbid $\mathrm{CD}$, were tested on the standardized Brain Resource International Database protocols and were compared with a group of matched healthy controls. Testing included a forced-choice facial emotion recognition task, from which response accuracy and reaction time were recorded.

Results: ADHD (with and without comorbid CD) was distinguished from healthy controls by poorer recognition of negative facial expressions and a quicker reaction time to correctly identify angry facial expressions. However, ADHD alone was distinguished from both the healthy control and the combined ADHD and CD groups by poorer recognition of positive facial expressions and a longer response reaction time those that were correctly identified. Notably, the combined ADHD and CD group was not differentiated from healthy controls by either accuracy or reaction time to positive facial expressions. Conclusions: ADHD with combined CD comprises a diagnostic group with emotion-related cognitive deficits that are distinct from those showed in ADHD alone and indicate a difference in the causal mechanisms underlying social cognition and emotion recognition deficits between these groups. This difference underscores the relevance of taking comorbidity into account in addressing both emotional awareness and social and peer relationship difficulties in ADHD.

\section{Do religious beliefs help us cope with traumatic experiences?}

\section{R Parslow}

Orygen Research Centre, The University of Melbourne, Melbourne, Australia

Background: It is widely assumed that holding spiritual beliefs offers individuals comfort and support in times of difficulty. It might be expected then that individuals who feel that religion is important to them and offers them strength and comfort would report being less affected after having been exposed to a potentially life-threatening trauma.

Methods: From 1999 to 2002, 7485 residents in Canberra and surrounds were interviewed for the PATH Through Life Project, a longitudinal survey being conducted by the Centre for Mental Health Research. Participants, drawn from three age groups, were reinterviewed from 2003 to 2006. Questions asked in both interviews covered sociodemographic, physical and mental health measures, and personality attributes. In both interviews, participants were also asked about their attendance at religious services, their position concerning religion and the extent to which religion was a source of strength and comfort to them.

In January 2003 before commencement of wave 2 interviews, a major bushfire hit the Canberra region. When reinterviewed, participants were asked about their level of exposure to the fire and the extent to which they experienced symptoms of post-traumatic stress. About $89.6 \%$ of wave 1 participants took part in wave 2, and of these, $6596(88.1 \%)$ answered questions concerning bushfire exposure.

Results: The analyses examined whether individuals with strong religious beliefs experienced lower levels of post-traumatic stress after the fires, compared with their less religious counterparts. Associations between these factors were found to vary across the life span and were strongest for participants in midlife. 